\title{
ABO blood group system and risk of positive surgical margins in patients treated with robot-assisted radical prostatectomy: results in 1114 consecutive patients
}

\author{
Antonio Benito Porcaro ${ }^{1}$ (1) Nelia Amigoni ${ }^{1}$ - Filippo Migliorini ${ }^{1} \cdot$ Riccardo Rizzetto $^{1}$ - Alessandro Tafuri ${ }^{1,3}$. \\ Pierluigi Piccoli ${ }^{4}$. Leone Tiso ${ }^{1}$. Clara Cerrato ${ }^{1}$. Alberto Bianchi ${ }^{1} \cdot$ Sebastian Gallina ${ }^{1} \cdot$ Rossella Orlando $^{1}$. \\ Mario De Michele ${ }^{1}$ - Alessandra Gozzo ${ }^{1}$. Stefano Zecchini Antoniolli ${ }^{1} \cdot$ Vincenzo De Marco $^{1}$ - Matteo Brunelli ${ }^{5}$. \\ Maria Angela Cerruto ${ }^{1} \cdot$ Walter Artibani $^{1} \cdot$ Salvatore Siracusano ${ }^{1} \cdot$ Alessandro Antonelli $^{2}$
}

Received: 30 March 2021 / Accepted: 9 June 2021 / Published online: 29 June 2021

(c) The Author(s) 2021

\begin{abstract}
To test the hypothesis of associations between the ABO blood group system (ABO-bg) and prostate cancer (PCa) features in the surgical specimen of patients treated with robot-assisted radical prostatectomy (RARP). Between January 2013 and October 2020, 1114 patients were treated with RARP. Associations of ABO-bg with specimen pathological features were evaluated by statistical methods. Overall, 305 patients were low risk (27.4\%), 590 intermediate risk (50\%) and 219 high risk (19.6\%). Pelvic lymph node dissection was performed in 678 subjects (60.9\%) of whom 79 (11.7\%) had cancer invasion. In the surgical specimen, tumor extended beyond the capsule in $9.8 \%$ and invaded seminal vesicles in $11.8 \%$ of cases. Positive surgical margins (PSM) were detected in 271 cases (24.3\%). The most frequently detected blood groups were A and O, which were equally distributed for both including 467 patients (41.9\%), followed by groups B (127 cases; 11.4\%) and AB (53 subjects; 4.8\%). Among specimen factors, the ABO-bgs associated only with the risk of PSM, which was higher for blood group O (30.4\%) compared with group A (19.5\%) after adjusting for other standard clinical predictors (odds ratio, $\mathrm{OR}=1.842 ; 95 \%$ CI 1.352-2.509; $p<0.0001$ ). Along the ABO-bgs, the risk of PSM was increased by group O independently by other standard preoperative factors. The ABO-bgs may represent a further physical factor for clinical assessment of PCa patients, but confirmatory studies are required.
\end{abstract}

Keywords Prostate cancer · Robot-assisted radical prostatectomy $\cdot$ ABO blood system $\cdot$ Tumor load $\cdot$ Tumor stage . Positive surgical margins

Antonio Benito Porcaro, Nelia Amigoni and Filippo Migliorini have contributed equally to this manuscript.

Antonio Benito Porcaro

drporcaro@yahoo.com

$\triangle$ Alessandro Tafuri

tafuri.alessandro@gmail.com

1 Department of Urology, Azienda Ospedaliera Universitaria Integrata Verona, University of Verona, Piazzale Stefani 1, 37126 Verona, Italy

2 Chairman, Department of Urology, Azienda Ospedaliera Universitaria Integrata, University of Verona, Verona, Italy

\section{Introduction}

Actually, prostate cancer $(\mathrm{PCa})$ is one of the most investigated cancers in the aging male who is likely to have the disease detected at early stages $[1,2]$. In early PCa, several

3 Department of Neuroscience, Imaging and Clinical Sciences, "G. D’Annunzio" University, Chieti, Italy

4 Department of Transfusion Medicine, Azienda Ospedaliera Universitaria Integrata, University of Verona, Verona, Italy

5 Department of Pathology, Azienda Ospedaliera Universitaria Integrata, University of Verona, Verona, Italy 
management options are proposed, which include active surveillance, primary radiation, and radical prostatectomy (RP), which may be performed by the open approach (ORP) or more frequently by the robot-assisted procedure (RARP), as well $[1,2]$. However, clinical PCa includes a heterogeneous set of patients who are classified into risk categories by prognostic clinical factors including prostate-specific antigen (PSA), tumor stage, and grade, as well [1, 2]. In the surgical specimen, tumor upgrading and upstaging as well as the detection of positive surgical margins (PSM) are unfavorable outcomes requiring further management decisions. So far, aggressive PCa biology may be detected in the surgical specimen after RARP; as such, further clinical factors are required to stratify risk categories $[1,2]$.

Potential preoperative factors for stratifying PCa clinical risk classes could be represented by blood group antigens, which are polymorphic, inherited structural characters that are present on the outer surface of the red cell membrane and are located on proteins, glycoproteins or glycolipids; furthermore, human blood group antigens have also been associated with clinical disorders[3]. The ABO blood group system (ABO-bgs) is the most important not only for being the first one discovered but also for both blood transfusions and organ transplantation; furthermore, it associates with non-oncological and oncological diseases. In case-control studies, the ABO-bgs has been associated with the risk of several epithelial cancers [4-10]. For example, the risk of gastric cancer was increased by blood group A while individual belonging to the non-O-bgs showed an increased risk of pancreatic cancer, as well [4-7]. Recently, associations between phenotype ABO-bgs and prostate cancer have been hypothesized by a case-control study that did not show any significant association [11]. However, a retrospective study, which investigated a small heterogeneous cohort of $\mathrm{PCa}$ patients, has shown associations between high-risk $\mathrm{PCa}$ and ABO-bgs [12]. Furthermore, another retrospective study showed that the ABO-bgs correlated with survival on $\mathrm{PCa}$ vaccine therapy [13]. So far, the hypothesis of associations between ABO-bgs and PCa is an unsettled topic that needs more clinical investigations, which should be interpreted according to the complexity of clinical and pathological manifestations of PCa [14].

In this study, we wanted to investigate associations between the ABO-bgs and PCa features in the surgical specimen of patients treated with RARP.

\section{Materials and methods}

\section{Study population}

The study was retrospective and approved by the internal Institutional Review Board. Informed signed consent was obtained by all patients. Data were collected prospectively but evaluated retrospectively. In a period ranging from January 2013 to October 2020, 1114 consecutive patients who underwent RARP were included after excluding cases who were under androgen blockade and/or had prior treatments for PCa. Surgical procedures were performed by five skilled and dedicated surgeons of whom two were classified as high volume. Clinical features including age (years), body mass index (BMI; $\left.\mathrm{kg} / \mathrm{m}^{2}\right)$, PSA $(\mathrm{ng} / \mathrm{mL})$, prostate volume (PV, $\mathrm{mL}$ ) and biopsy positive cores (BPC; \%) were evaluated. Tumors were staged according to clinical and pathological TNM system [1, 2]. RARP was eventually associated with pelvic lymph node dissection (PLND) according to guideline recommendations or tumor upgrading probability for the low-risk category $[15,16]$. Lymph node dissection was developed according to a standard anatomical template including external iliac, obturator, Cloquet's and Marcille's regions $[17,18]$. Since January 2017 , our policy is not to place a drain in the pelvic cavity independently by performing or not an extended PLND [19].

Operations were performed by surgeons who were classified into high- and low-volume ( $>100$ ) according to study reporting a reduction PSM rate after 100 cases were performed [20]. Specimens were evaluated for tumor grade and stage, surgical margins, number of removed, and metastatic lymph nodes. Tumors were graded according to the International Society of Urological Pathology (ISUP) system [1, 2]. Preoperative surgical risk was evaluated by the American Society of Anesthesiologists (ASA) score system [21]. Postoperative surgical complications were graded according to the Clavien-Dindo system [1, 2]. At hospital discharge, patients were followed for a period of 90 days to detect complications and/or hospital readmission events. In each patient, the genotype ABO blood group system was assessed preoperatively by the Department of Transfusion Medicine. Blood groups were routinely determined on microplates by reactant and instrumentation LIFE (AstraFormedic, Gruppo De Mori).

\section{Statistical methods}

The hypothesis of associations between the ABO-bgs and PCa biology was tested on specimen pathological features. According to their distributions, continuous variables were represented as medians (interquartile range, IQR) while categorical variables were assessed as frequencies (percentages). The association of the ABO-bg system with clinical and pathological variables was assessed by the multinomial logistic regression model (univariate analysis). The independent association of the ABO-bgs with specimen pathological features was eventually assessed by the logistic regression model (univariate and multivariate analysis). The fit of potential multivariate models including the ABO-bgs 
after adjusting for $\mathrm{PCa}$ clinical features was assessed the Hosmer-Lemeshow test, which was performed after computing the associated decile contingency tables. The software used to run the analysis was IBM-SPSS version 26. All tests were two-sided with $p<0.05$ considered to indicate statistical significance.

\section{Results}

\section{Demographics and cancer features of the patient population}

Table 1 shows the demographics of the patient population that included 1114 cases of whom 305 were low-risk (27.4\%), 590 intermediate-risk (50\%) and 219 high-risk (19.6\%). Pelvic lymph node dissection was performed in 678 subjects $(60.9 \%)$ of whom $79(11.7 \%)$ had cancer invasion. In the surgical specimen, tumor extended beyond the gland in 240 patients (21.6\%) with extracapsular extension in 9.8\% and seminal vesicle invasion in $11.8 \%$ of cases, respectively; furthermore, surgical margins resulted involved by cancer in 271 cases (24.3\%). PMS location was at apex in $41 \%$ of cases and in $34 \%$ at posterolateral base gland (left or right).

Preoperative physical status included prevalently ASA grade group II (905 cases; 81.2\%) whereas groups I and III almost overlapped for including 104 (9.3\%) and 105 (9.5\%) cases, respectively. As shown in Fig. 1, the most frequently detected blood groups were $\mathrm{A}$ and $\mathrm{O}$, which were equally distributed for both being detected in 467 patients (41.9\%); furthermore, groups B (127 cases; 11.4\%) and AB (53 subjects; $4.8 \%$ ) then followed. Other clinical and perioperative features are detailed in the referred table.

Most part of the procedures was performed by high-volume surgeons and no significant differences in PSM rate were found according to surgeon experience.

\section{Associations of ABO-bg system with specimen pathological features}

Table 2 summarizes the results of potential associations of the ABO-bg with clinical, pathological, and perioperative features of the investigated patient population. For the overlapping distribution of the two main systems, blood groups $\mathrm{O}, \mathrm{B}$, and $\mathrm{AB}$ were compared with group $\mathrm{A}$. Overall, the risk of detecting PSM was significantly higher for blood group O compared with group A (odds ratio, $\mathrm{OR}=1.805 ; 95 \% \mathrm{CI}$ $1.355-2.442 ; p<0.0001)$. Figure 2 depicts the distribution of PSM in each group. Excluding age, which was inversely related in blood group B when compared with group A, no other significant associations were detected.

\section{The ABO-bg system as an independent predictor of the risk of PSM}

On multivariate analysis, the risk of PSM still associated with blood group $\mathrm{O}$ when compared with group A (odds ratio, $\mathrm{OR}=1.820 ; 95 \%$ CI $1.344-2.464 ; p<0.0001)$ independently by BMI (inverse association) for physical factors at clinical presentation, as shown in Table 3. As expected, the risk of PSM also associated with clinical and pathological PCa features. The risk of PSM increased as PSA, BPC, pT and pathological tumor grade increased as well decreased as TV increased. Table 4 shows the risk of PSM as predicted by preoperative multivariate models including the ABO-bgs, which was adjusted for BMI in model I as well as for PCa features in model II (PSA, BPC, PV). The risk of detecting PSM in the surgical specimen was higher for blood group O (30.4\%) compared with group A (19.5\%) with the former increasing the predictive power from model I $(\mathrm{OR}=1.820$; 95\% CI 1.344-2.464; $p<0.0001)$ to model II $(\mathrm{OR}=1.842$; 95\% CI $1.352-2.509 ; p<0.0001)$, as shown by the Hosmer-Lemeshow test and associated contingency tables.

\section{Discussion}

The ABO-bgs is traced out by the $\mathrm{ABO}$ gene, which is single and located on chromosome 9q34; furthermore, it still remains the most important system for both transfusion and transplantation medicine [3, 4]. The ABO-bgs has been associated with the risk of several carcinomas such as stomach, pancreas, ovary, kidney, and skin [4-12]. Specifically, it has been demonstrated that genotype blood group non-O increased the risk of cancers involving pancreas, kidney, and ovary but not non-melanoma skin cancer, which was instead increased by group $\mathrm{O}$; furthermore, gastric cancer was the first malignant tumor that associated with phenotype A-bgs [3-12]. So far, several studies show associations between the ABO-bgs and epithelial cancers. So far, a potential association between the ABO-bgs and PCa could be supposed. Indeed, $\mathrm{PCa}$ shows complex clinical and pathological manifestations that should be considered when planning and analyzing clinical studies [14]. Potential associations of the ABO-bgs with PCa represents a new topic, which is actually in progress. In a large case-control study, Iodice et al. did not show any significant association between the ABObgs and risk of epithelial cancers, which also included PCa; furthermore, in that trial, the distribution of the ABO-bg between controls vs PCa case was $46 \%$ vs $42 \%$ for group $\mathrm{O}$, $42 \%$ vs $43 \%$ for group A, $9 \%$ vs $10 \%$ for group B and $3 \%$ vs $4 \%$ for group AB [4]. Markt et al. did not find any significant association between $\mathrm{ABO}-\mathrm{bgs}$ and risk of aggressive $\mathrm{PCa}$ or PCa specific mortality in a large case-control study, which was restricted to men of European ancestry, including 2774 
Table 1 Demographics of the prostate cancer population $(n=1114)$ that was treated with robot-assisted radical prostatectomy (RARP)

\begin{tabular}{|c|c|}
\hline & $\begin{array}{l}\text { Median (IQR) or } \\
\text { frequency (\%) }\end{array}$ \\
\hline \multicolumn{2}{|l|}{ Clinical factors } \\
\hline Age (years) & $65(61-70)$ \\
\hline Body mass index, BMI $\left(\mathrm{kg} / \mathrm{m}^{2}\right)$ & $25.7(23.9-28)$ \\
\hline Prostate specific antigen, PSA $(\mu \mathrm{g} / \mathrm{L})$ & $7(5.1-9.7)$ \\
\hline Prostate volume, PV (mL) & $40(30.3-52)$ \\
\hline Biopsy positive cores, BPC (\%) & $34.5(21-53)$ \\
\hline \multicolumn{2}{|c|}{ International Society of Urologic Pathology (ISUP) tumor grade system } \\
\hline ISUP $=1$ & $436(39.1)$ \\
\hline ISUP $=2$ & $356(32.0)$ \\
\hline ISUP $=3$ & $192(17.2)$ \\
\hline ISUP $=4$ & $106(9.5)$ \\
\hline ISUP $=5$ & $24(2.2)$ \\
\hline \multicolumn{2}{|l|}{ Tumor clinical stage (cT) } \\
\hline $\mathrm{cT} 1$ & $687(61.7)$ \\
\hline $\mathrm{cT} 2 / 3$ & $427(38.3)$ \\
\hline \multicolumn{2}{|l|}{ Clinical nodal stage $(\mathrm{cN})$} \\
\hline $\mathrm{cNO}$ & $1058(95)$ \\
\hline $\mathrm{cN} 1$ & $56(5)$ \\
\hline \multicolumn{2}{|c|}{ American Society of Anesthesiologists' (ASA) physical system } \\
\hline ASA I & $104(9.3)$ \\
\hline ASA II & $905(81.2)$ \\
\hline ASA III & $105(9.5)$ \\
\hline \multicolumn{2}{|l|}{ D’Amico risk groups } \\
\hline Low risk class & $305(27.4)$ \\
\hline Intermediate risk class & $590(53.0)$ \\
\hline High risk class & $219(19.6)$ \\
\hline \multicolumn{2}{|l|}{ Pathological factors } \\
\hline Prostate weight; gr (PW) & $51(42-65)$ \\
\hline ISUP $=1$ & $143(12.8)$ \\
\hline ISUP $=2$ & $438(39.3)$ \\
\hline ISUP $=3$ & $303(27.2)$ \\
\hline $\mathrm{ISUP}=4$ & $158(14.2)$ \\
\hline ISUP $=5$ & $72(6.5)$ \\
\hline \multicolumn{2}{|l|}{ Pathological tumor stage (pT) } \\
\hline pT2 & $874(78.5)$ \\
\hline pT3a & $109(9.8)$ \\
\hline pT3b & $131(11.8)$ \\
\hline \multicolumn{2}{|l|}{ Positive surgical margin (PSM) } \\
\hline No & $843(75.7)$ \\
\hline Yes & $271(24.3)$ \\
\hline \multicolumn{2}{|l|}{ Pathological nodal staging (pN) } \\
\hline pNO & $599(53.8)$ \\
\hline pN1 & $79(7.1)$ \\
\hline $\mathrm{pNx}$ & $436(39.1)$ \\
\hline Lymph nodes removed (number) & $25(20-32)$ \\
\hline \multicolumn{2}{|l|}{ Perioperative factors } \\
\hline High volume surgeon (HVS) & $600(53.9)$ \\
\hline Low volume surgeon (LVS) & $475(42.6)$ \\
\hline Unknown & $39(3.5)$ \\
\hline Operating time; $\min (\mathrm{OT})$ & $233(205-259.3)$ \\
\hline
\end{tabular}


Table 1 (continued)

\begin{tabular}{lc}
\hline & $\begin{array}{c}\text { Median (IQR) or } \\
\text { frequency (\%) }\end{array}$ \\
\hline Blood lost; mL (BL) & $300(150-400)$ \\
Any post-operative Clavien-Dindo complication at discharge (CDC) & $273(24.5)$ \\
Length of hospital stay; days (LOHS) & $4(4-5)$ \\
Hospital readmission; $n(\%)$ & $35(3.1)$ \\
\hline
\end{tabular}

$I Q R$ interquartile range, $\%$ percentage

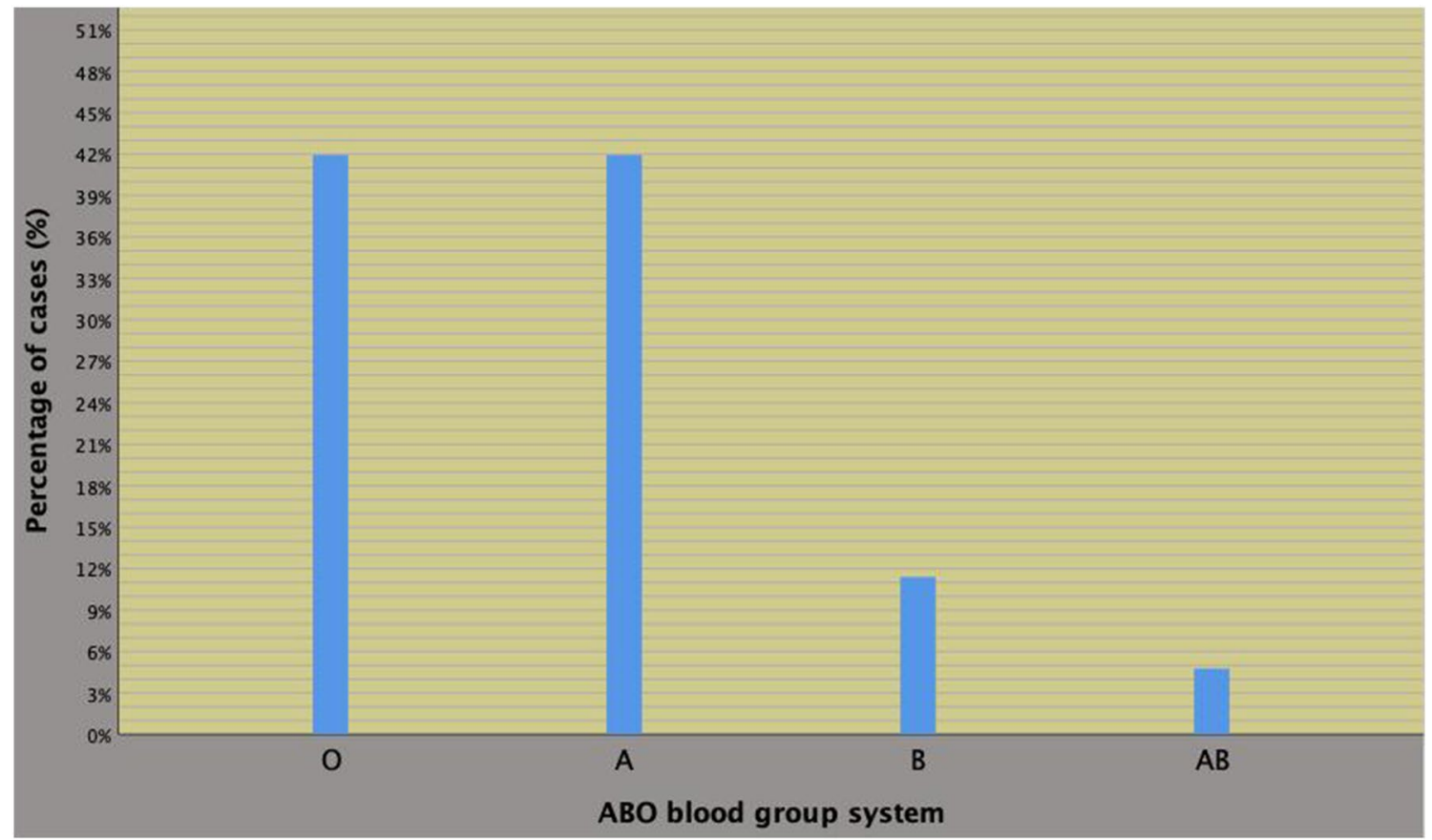

Fig. 1 Distribution of the ABO blood group system in 1114 prostate cancer $(\mathrm{PCa})$ subjects who underwent robot-assisted radical prostatectomy (RARP). As shown, the most frequently detected blood

aggressive PCa cases and 4443 controls; furthermore, the distribution of the ABO-bgs for controls versus cases was $42 \%$ vs $40 \%$ for group O, $43 \%$ vs $44 \%$ for group A, $10 \%$ vs $12 \%$ for group B and 5\% vs 5\% for group AB [11]. Multhana et al., in a retrospective analysis of prospective phase II trial on immunotherapies in PCa (PROSTVAC-VF), showed longer median survival in patients with blood type $\mathrm{B}$ and $\mathrm{O}$ compared with groups $\mathrm{A}$ and $\mathrm{AB}$ [13]. Wang et al., in a single-center study conducted on the Chinese PCa population, showed that the risk of aggressive $\mathrm{PCa}$ was higher for non-O blood groups compared with group $\mathrm{O}$; however, the trial had several limitations for being retrospective, for the size of the sample, for the definition of "high-risk" patients who were widely heterogenous for being at the same time high-risk, locally advanced or even metastatic; furthermore, the low-middle risk subpopulation included only 43 (18.1\%); as such, the results of the study are difficult to apply to the groups were $\mathrm{A}$ and $\mathrm{O}$, which were equally distributed for both being detected in 467 patients (41.9\%), followed by groups B (127 cases; $11.4 \%)$ and $\mathrm{AB}$ (53 subjects; $4.8 \%)$

Caucasian population [12]. In a Caucasian PCa population treated with RARP, we found out an independent association between the ABO-bgs and risk of PSM in the surgical specimen. Specifically, the risk of detecting a PSM was higher for blood group $\mathrm{O}$ when compared with group $\mathrm{A}$, independently by physical (BMI) and cancer clinical features (PSA, BPC and PV). We have also shown that the ABO-bgs was an effective predictor of PSM after adjusting for physical and cancer preoperative factors thus demonstrating close association with PCa biology. As such, the results of our study represent a novelty in the literature dealing with this subject and might have implications in clinical practice.

Actually, the prevalence of PSM after RP ranges from 8.8 to $37 \%$; and independent predictors are represented by surgeon's volume and tumor biology including features related to load, extension and aggressiveness of cancer [22-24]. In tertiary referral centers, RARP is the most prevalent 
Table 2 Associations of clinical, pathological, and perioperative factors with the ABO blood group system in 1114 prostate cancers patients treated with robot assisted radical prostatectomy (univariate analysis)

\begin{tabular}{|c|c|c|c|c|c|c|}
\hline \multirow[t]{2}{*}{ Statistics } & \multicolumn{2}{|l|}{ Blood group O vs A } & \multicolumn{2}{|l|}{ Blood group B vs A } & \multicolumn{2}{|l|}{ Blood group $\mathrm{AB}$ vs $\mathrm{A}$} \\
\hline & OR $(95 \% \mathrm{CI})$ & $p$ value & OR $(95 \% \mathrm{CI})$ & $p$ value & OR (95\% CI) & $p$ value \\
\hline Age & $0.984(0.965-1.004)$ & 0.115 & $0.968(0.940-0.997)$ & 0.033 & $0.979(0.938-1.022)$ & 0.331 \\
\hline BMI & $1.011(0.970-1.053)$ & 0.610 & $1.015(0.954-1.079)$ & 0.648 & $0.926(0.844-1.017)$ & 0.109 \\
\hline PSA & $1.006(0.989-1.024)$ & 0.484 & $1.000(0.972-1.029)$ & 1.000 & $1.000(0.960-1.042)$ & 1.000 \\
\hline PV & $0.997(0.990-1.004)$ & 0.431 & $0.997(0.986-1.008)$ & 0.586 & $0.999(0.984-1.015)$ & 0.929 \\
\hline $\mathrm{BPC}$ & $0.998(0.992-1.004)$ & 0.522 & $0.998(0.989-1.008)$ & 0.747 & $0.997(0.984-1.010)$ & 0.647 \\
\hline ISUP $<3$ & Ref & & Ref & & Ref & \\
\hline ISUP $>2$ & $0.969(0.730-1.287)$ & 0.828 & $1.001(0.650-1.541)$ & 0.998 & $1.052(0.556-1.955)$ & 0.871 \\
\hline $\mathrm{cT}<2$ & Ref & & Ref & & Ref & \\
\hline $\mathrm{cT}>1$ & $0.904(0.694-1.179)$ & 0.904 & $1.070(0.717-1.597)$ & 0.741 & $1.656(0.937-2.927)$ & 1.656 \\
\hline cN0 & Ref & & Ref & & Ref & \\
\hline $\mathrm{cN} 1$ & $1.371(0.758-2.482)$ & 0.296 & $1.108(0.435-2.821)$ & 0.829 & $1.341(0.385-4.672)$ & 0.645 \\
\hline PW & $0.998(0.991-1.005)$ & 0.559 & $0.996(0.986-1.007)$ & 0.985 & $1.006(0.993-1.020)$ & 0.365 \\
\hline ISUP $<3$ & Ref & & Ref & & Ref & \\
\hline ISUP $>2$ & $1.118(0.865-1.445)$ & 0.395 & $0.930(0.625-1.379)$ & 0.718 & $1.186(0.672-2.094)$ & 0.556 \\
\hline pT2 & Ref & & Ref & & Ref & \\
\hline pT3a & $1.099(0.714-1.693)$ & 0.667 & $0.781(0.381-1.602)$ & 0.500 & $1.171(0.472-2.909)$ & 0.733 \\
\hline pT3b & $1.186(0.799-1.762)$ & 0.397 & $0.796(0.410-1.544)$ & 0.499 & $0.829(0.314-2.188)$ & 0.705 \\
\hline No PSM & Ref & & Ref & & Ref & \\
\hline PSM & $1.805(1.355-2.442)$ & $<0.0001$ & $1.169(0.725-1.885)$ & 0.523 & $0.961(0.465-1.984)$ & 0.914 \\
\hline pNO & Ref & & Ref & & Ref & \\
\hline pN1 & $0.918(0.542-1.555)$ & 0.751 & $1.328(0.617-2.858)$ & 0.468 & $2.425(0.962-6.114)$ & 0.060 \\
\hline $\mathrm{LN}(n)$ & $1.003(0.987-1.019)$ & 0.750 & $1.015(0.991-1.040)$ & 0.231 & $0.994(0.957-1.034)$ & 0.776 \\
\hline No PLND & Ref & & Ref & & Ref & \\
\hline PLND & $1.201(0.921-1.556)$ & 0.177 & $0.806(0.542-1.197)$ & 0.285 & $0.856(0.482-1.519)$ & 0.595 \\
\hline OT & $1.001(0.999-1.003)$ & 0.362 & $0.998(0.994-1.002)$ & 0.269 & $0.999(0.994-1.004)$ & 0.667 \\
\hline BL & $1.000(1.000-1.000)$ & 0.715 & $1.000(0.999-1.000)$ & 0.476 & $1.000(0.998-1.001)$ & 0.425 \\
\hline $\mathrm{CDS}=0$ & Ref & & Ref & & Ref & \\
\hline $\mathrm{CDS}>0$ & $1.123(0.033-1.514)$ & 0.446 & $1.201(0.768-1.878)$ & 0.423 & $0.860(0.428-1.728)$ & 0.672 \\
\hline LOHS & $1.039(0.971-1.111)$ & 0.265 & $0.918(0.800-1.052)$ & 0.219 & $1.048(0.918-1.198)$ & 0.488 \\
\hline No RAD & Ref & & Ref & & Ref & \\
\hline RAD & $1.258(0.583-2.718)$ & 0.559 & $1.880(0.691-5.112)$ & 0.216 & $1.487(0.324-6.830)$ & 0.610 \\
\hline
\end{tabular}

See also Table 1

$O R$ odds ratio, $C I$ confidence interval

procedure and it decreases the risk of PSM when compared with ORP; as a result, these outcomes further support the advantages of oncological outcomes of robotic surgery [25]. We did not find difference in other clinical pathological features among ABO-bgs groups. However, we have confirmed that clinical and pathological features related to the risk of PSM, which increased as PSA, BPC, tumor upstaging, and upgrading after surgery. The small number of cases including blood groups $\mathrm{B}$ and $\mathrm{AB}$ may explain the missed association with the risk of PSM for these subgroups. Additionally, no differences were found according to surgeons' experience in the PSM rate. This data could be influenced by the high number of procedures performed by high-volume surgeons compared to low-volume surgeons, and maybe in contrast with our previous experience where we included only one high-volume surgeon according to the department organization referred to that cohort time [26, 27].

Furthermore, our study shows that, over clinical cancer features, also physical factors including BMI and ABO-bgs predicted the risk of PSM in multivariate models, as well. This is an important issue when counseling PCa patients for RARP because the detection of PSM represents an unfavorable pathological outcome that adversely impacts the natural history of the disease for biochemical recurrence, metastatic progression, and disease-specific mortality [20]. Additionally, it is known that obese patients represent a special 


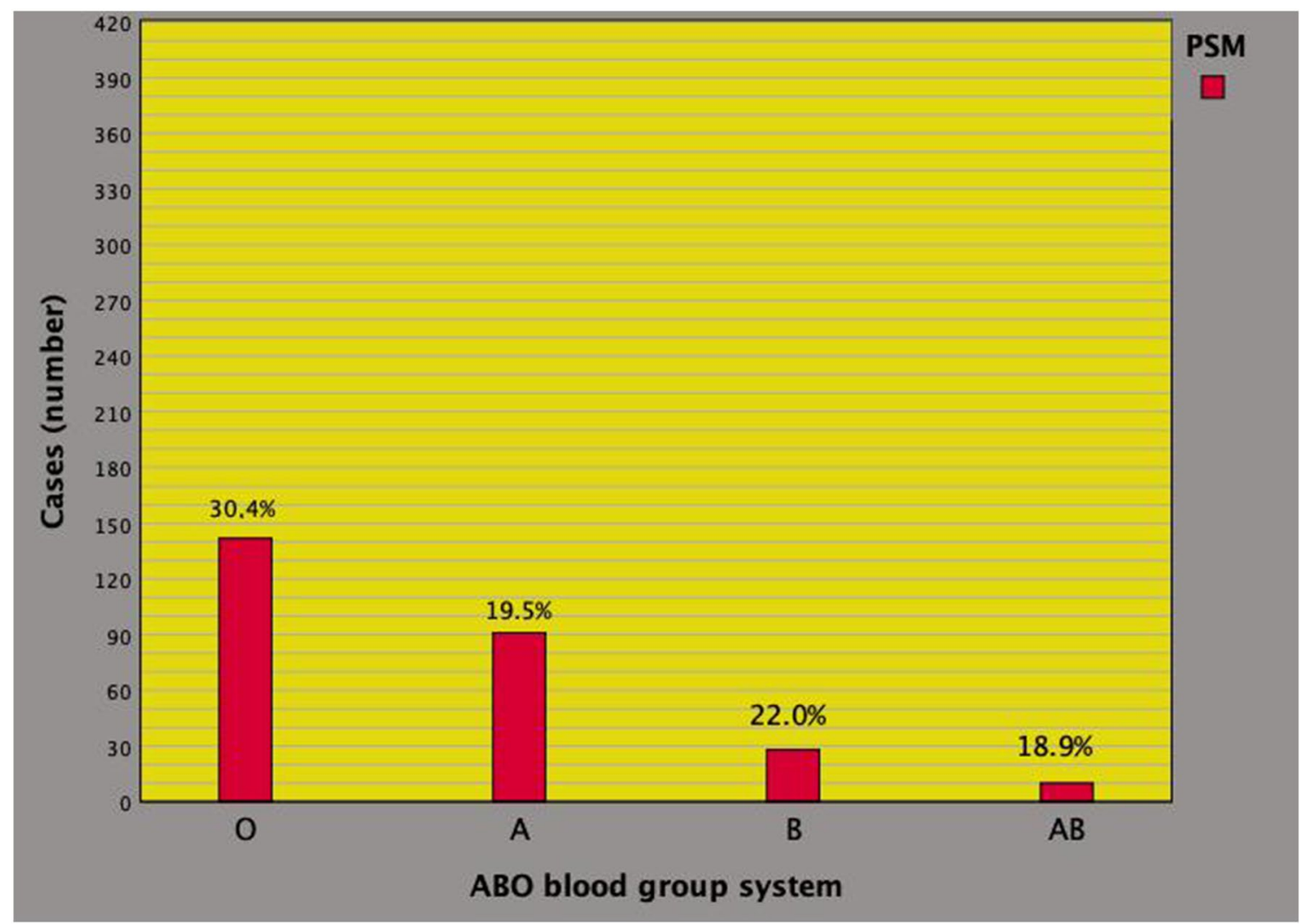

Fig. 2 Distribution of positive surgical margins (PSM) along the ABO blood group system in the surgical specimen of 1114 consecutive prostate cancer ( $\mathrm{PCa}$ ) who were treated with robot-assisted radi-

category that associates with a more challenging surgery with the increased risk of less accurate oncological surgery and increased risk of cancer recurrence and progression after open and or robotic procedures [20,28-32].

The results of our study might be explained by considering biology of the ABO-bg system that has antigens expressed not only on erythrocytes, but also on epithelial and endothelial cells, as well [3-12]. Such association has been explained by several mechanisms including intercellular adhesion, membrane signaling, angiogenesis, inflammation, and immune surveillance of malignant transformed cells; furthermore, the system may also be related to tumor progression [3-12]. As a theory, the expression of genotype $\mathrm{O}$ on $\mathrm{PCa}$ cells may influence intercellular adhesion and membrane signaling; furthermore, stimulation of angiogenesis as decreased immunosurveillance may promote tumor growth and extension beyond the prostate capsule thus increasing the risk of PSM.

This study has limitations for being retrospective and for not adjusting for nerve-sparing surgery because such data were not available for all patients. Additionally, data cal prostatectomy (RARP). As illustrated, the distribution of cases was as follows: 142 (30.4\%) for group O, 91 (19.5\%) for group A, 28 (22\%) for group B, and 10 (18.9\%) for group $\mathrm{AB}$

on tumor location were not available. However, it has also strengths for data being collected prospectively and for the population being large and homogenous; additionally, blood groups were all determined at the Department of Transfusion Medicine of our hospital. Furthermore, we have already shown that the risk of biochemical recurrence associated with focal PSM, which closely related to the high-volume surgeon, while nerve-sparing surgery did not have any significant impact [26, 27, 33-35]. As such, our study has also clinical implications for ABO-bgs being an independent predictor of PSM risk.

\section{Conclusions}

Along the ABO-bgs, the risk of PSM was increased by group $\mathrm{O}$ independently by other standard preoperative factors. The ABO-bgs may represent a further physical factor for clinical assessment of PCa patients, but confirmatory studies are required. 
Table 3 Associations of physical, cancer and perioperative factors with the risk of positive surgical margins (PSM) in 1114 prostate cancer patients treated with robot assisted radical prostatectomy (RARP)

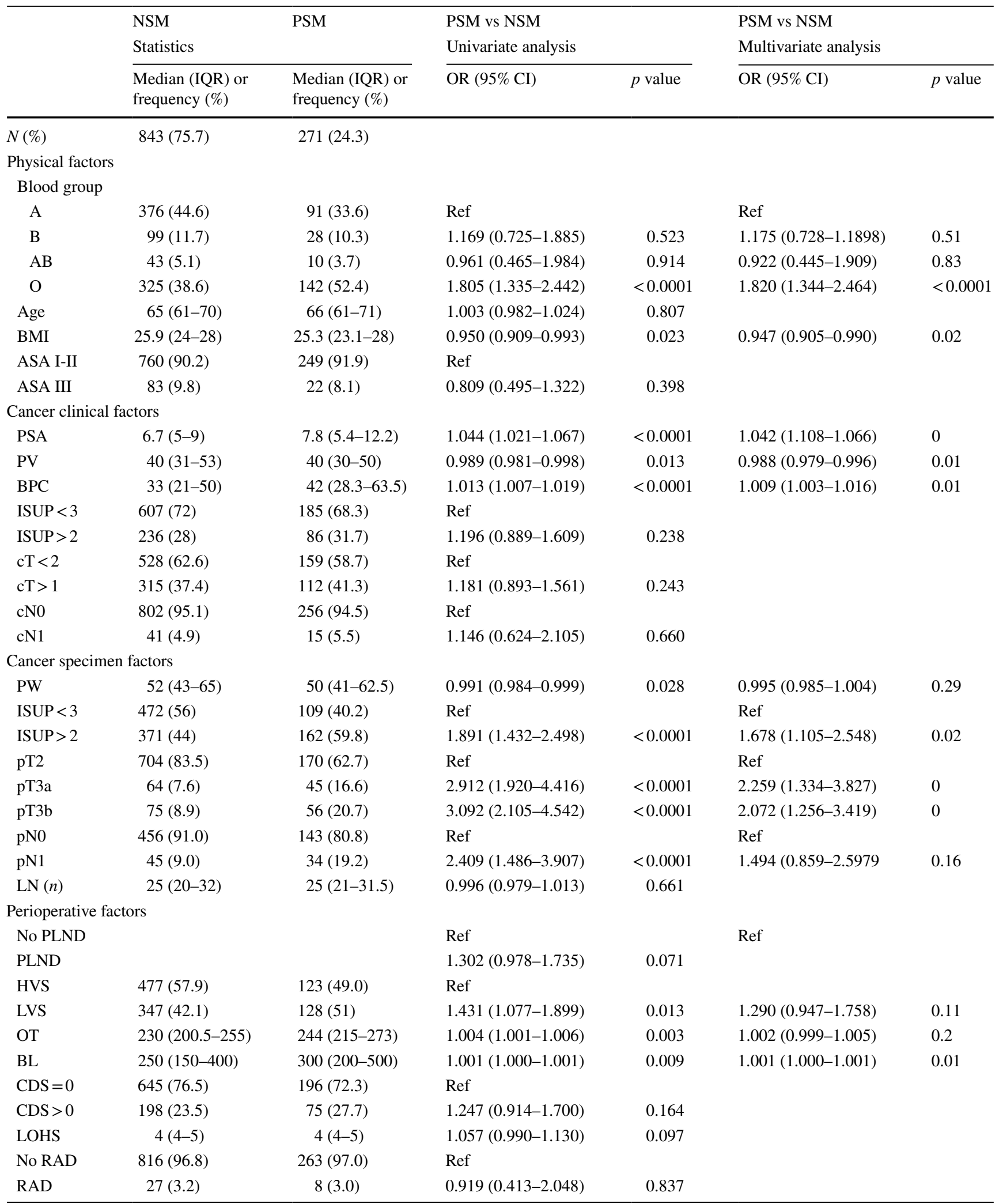

See also Table 1

$N S M$ negative surgical margins, $I Q R$ interquartile range, $O R$ odds ratio, $C I$ confidence interval 
Table 4 Multivariate clinical models of ABO blood group system predicting the risk of positive surgical margins (SM) in 1114 prostate cancer patients treated with robot-assisted radical prostatectomy (RARP)

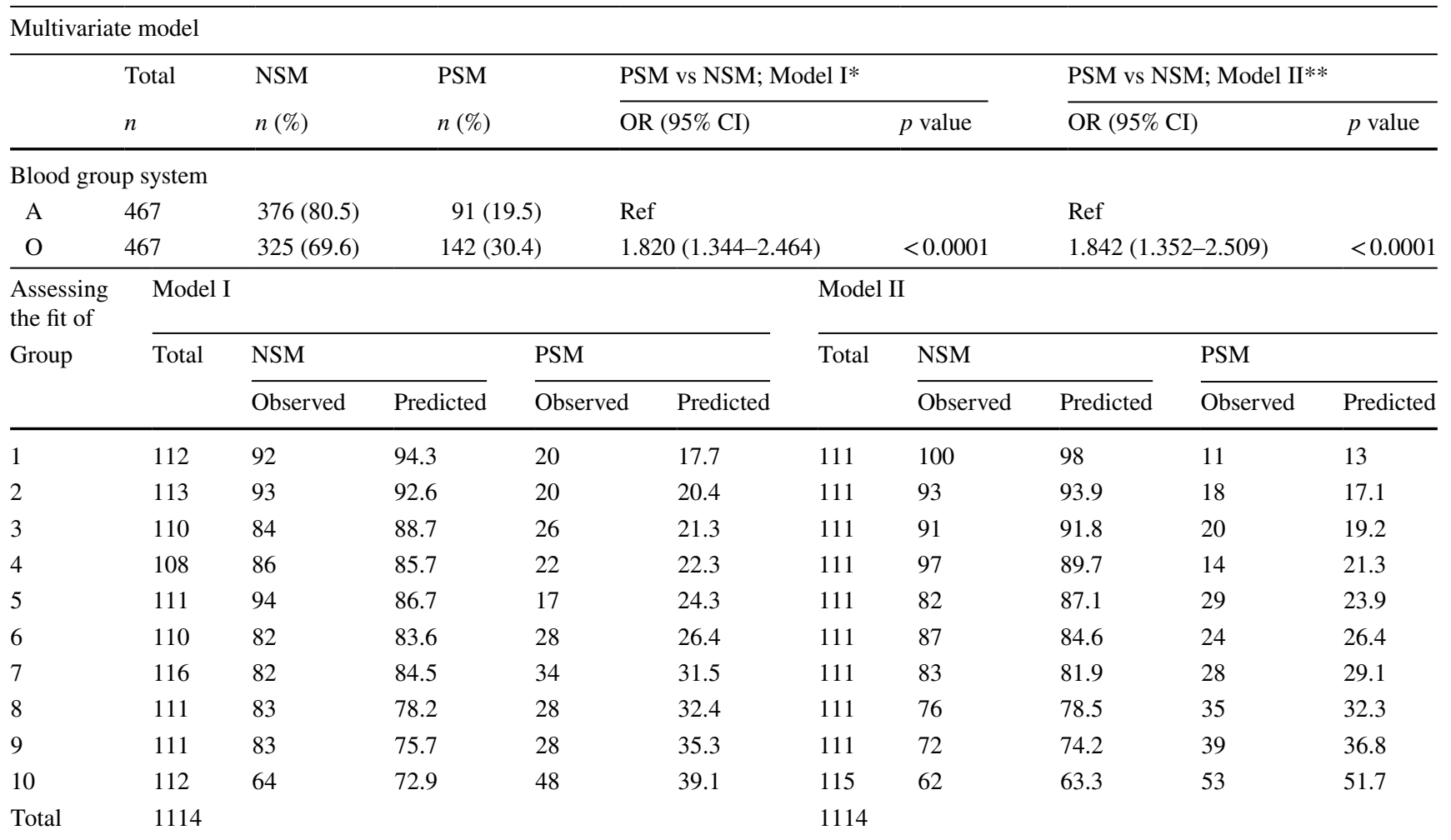

See also Table 1. Test of Hosmer-Lemeshow: (a) Model I: Chi-squared 11.109; degree freedom =8; $p=0.196$, overall accuracy 75.7\%; (b) Model II: Chi-squared 5.842, degree freedom $=8, p=0.665$, overall accuracy $76.1 \%$

$N S M$ negative SM, PSM positive SM, OR odds ratio, $C I$ confidence interval

*Model adjusted for blood group B, blood group AB and BMI

**Model adjusted for blood group B, blood group AB, BMI, PSA, PV and BPC

Funding Open access funding provided by Università degli Studi di Verona within the CRUI-CARE Agreement.

\section{Declarations}

Conflict of interest The authors declare that they have no conflict of interest.

Ethical approval All procedures performed in studies involving human participants were in accordance with the ethical standards of the institutional and/or national research committee and with the 1964 Helsinki Declaration and its later amendments or comparable ethical standards.

Informed consent Informed consent was obtained from all individual participants included in the study.

Open Access This article is licensed under a Creative Commons Attribution 4.0 International License, which permits use, sharing, adaptation, distribution and reproduction in any medium or format, as long as you give appropriate credit to the original author(s) and the source, provide a link to the Creative Commons licence, and indicate if changes were made. The images or other third party material in this article are included in the article's Creative Commons licence, unless indicated otherwise in a credit line to the material. If material is not included in the article's Creative Commons licence and your intended use is not permitted by statutory regulation or exceeds the permitted use, you will need to obtain permission directly from the copyright holder. To view a copy of this licence, visit http://creativecommons.org/licenses/by/4.0/.

\section{References}

1. Mottet $\mathrm{N}$ et al (2020) EAU - ESTRO - ESUR - SIOG guidelines on prostate cancer 2020, in European Association of Urology Guidelines. 2020 Edition. 2020, European Association of Urology Guidelines Office: Arnhem, The Netherlands

2. Carroll PH, Mohler JL (2018) NCCN guidelines updates: prostate cancer and prostate cancer early detection. J Natl Compr Cancer Netw 16(5S):620-623

3. Reid ME, Bird GW (1990) Associations between human red cell blood group antigens and disease. Transfus Med Rev 4(1):47-55

4. Iodice $\mathrm{S}$ et al (2010) ABO blood group and cancer. Eur J Cancer 46(18):3345-3350

5. Wolpin BM et al (2010) Pancreatic cancer risk and ABO blood group alleles: results from the pancreatic cancer cohort consortium. Can Res 70(3):1015-1023 
6. Nakao $\mathrm{M}$ et al (2011) ABO blood group alleles and the risk of pancreatic cancer in a Japanese population. Cancer Sci 102(5):1076-1080

7. Risch HA et al (2010) ABO blood group, Helicobacter pylori seropositivity, and risk of pancreatic cancer: a case-control study. J Natl Cancer Inst 102(7):502-505

8. Joh H-K, Cho E, Choueiri TK (2012) ABO blood group and risk of renal cell cancer. Cancer Epidemiol 36(6):528-532

9. Gates MA et al (2011) ABO blood group and incidence of epithelial ovarian cancer. Int J Cancer 128(2):482-486

10. Xie J et al (2010) ABO blood group and incidence of skin cancer. PLoS ONE 5(8):e11972

11. Markt SC et al (2015) ABO blood group alleles and prostate cancer risk: results from the breast and prostate cancer cohort consortium (BPC3). Prostate 75(15):1677-1681

12. Wang F-M et al (2017) Association of ABO blood types and clinicopathological features of prostate cancer. Dis Markers 2017:9237481

13. Muthana SM et al (2015) ABO blood type correlates with survival on prostate cancer vaccine therapy. Oncotarget 6(31):32244

14. Giovannucci E et al (2007) Risk factors for prostate cancer incidence and progression in the health professionals follow-up study. Int J Cancer 121(7):1571-1578

15. Porcaro $\mathrm{AB}$ et al (2016) Low-risk prostate cancer and tumor upgrading to higher patterns in the surgical specimen. Analysis of clinical factors predicting tumor upgrading to higher Gleason patterns in a contemporary series of patients who have been evaluated according to the modified Gleason score grading system. Urol Int 97(1):32-41

16. Porcaro AB et al (2017) Clinical factors of disease reclassification or progression in a contemporary cohort of prostate cancer patients elected to active surveillance. Urol Int 98(1):32-39

17. Porcaro AB et al (2019) Lymph nodes invasion of Marcille's fossa associates with high metastatic load in prostate cancer patients undergoing extended pelvic lymph node dissection: the role of "marcillectomy." Urol Int 103(1):25-32

18. Cacciamani GE et al (2019) Extended pelvic lymphadenectomy for prostate cancer: should the Cloquet's nodes dissection be considered only an option? Minerva Urol Nefrol 71(2):136-145

19. Porcaro AB et al (2021) Is a drain needed after robotic radical prostatectomy with or without pelvic lymph node dissection? Results of a single-centerrandomized clinical trial. J Endourol 35(6):922-928

20. Yossepowitch $\mathrm{O}$ et al (2014) Positive surgical margins after radical prostatectomy: a systematic review and contemporary update. Eur Urol 65(2):303-313

21. Dripps RD, Lamont A, Eckenhoff JE (1961) The role of anesthesia in surgical mortality. JAMA 178(3):261-266

22. Fontenot PA, Mansour AM (2013) Reporting positive surgical margins after radical prostatectomy: time for standardization. BJU Int 111(8):E290-E299
23. Yossepowitch O et al (2009) Positive surgical margins in radical prostatectomy: outlining the problem and its long-term consequences. Eur Urol 55(1):87-99

24. Porcaro AB et al (2018) Positive association between preoperative total testosterone levels and risk of positive surgical margins by prostate cancer: results in 476 consecutive patients treated only by radical prostatectomy. Urol Int 101(1):38-46

25. Antonelli A et al (2017) Positive surgical margins and early oncological outcomes of robotic vs open radical prostatectomy at a medium case-load institution. Minerva Urol Nefrol 69(1):63-68

26. Porcaro AB et al (2019) High surgeon volume and positive surgical margins can predict the risk of biochemical recurrence after robot-assisted radical prostatectomy. Ther Adv Urol 11:1756287219878283

27. Porcaro AB et al (2019) Surgeon volume and body mass index influence positive surgical margin risk after robot-assisted radical prostatectomy: results in 732 cases. Arab J Urol 17(3):234-242

28. $\mathrm{Xu} \mathrm{MC}$ et al (2021) Obesity is associated with longer survival independent of sarcopenia and myosteatosis in metastatic and/or castrate-resistant prostate cancer. J Urol 205(3):800-805

29. Porcaro AB et al (2020) High body mass index predicts multiple prostate cancer lymph node metastases after radical prostatectomy and extended pelvic lymph node dissection. Asian J Androl 22(3):323

30. Gong $\mathrm{Z}$ et al (2007) Obesity is associated with increased risks of prostate cancer metastasis and death after initial cancer diagnosis in middle-aged men. Cancer Interdiscip Intl J Am Cancer Soc 109(6):1192-1202

31. Porcaro AB et al (2019) Body mass index is an independent predictor of Clavien-Dindo grade 3 complications in patients undergoing robot-assisted radical prostatectomy with extensive pelvic lymph node dissection. J Robot Surg 13(1):83-89

32. Branche B et al (2018) MP34-11 obesity, risk of biochemical recurrence, and PSADT after radical prostatectomy: results from the search database. J Urol 199(4S):e442-e442

33. Porcaro AB et al (2020) Risk factors of positive surgical margins after robot-assisted radical prostatectomy in high-volume center: results in 732 cases. J Robot Surg 14(1):167-175

34. Porcaro AB et al (2020) Linear extent of positive surgical margin impacts biochemical recurrence after robot-assisted radical prostatectomy in a high-volume center. J Robot Surg 14(4):663-675

35. Kin L et al (2011) Risk factors of positive surgical margin and biochemical recurrence of patients treated with radical prostatectomy: a single-center 10-year report. Chin Med J 124(7):1001-1005

Publisher's Note Springer Nature remains neutral with regard to jurisdictional claims in published maps and institutional affiliations. 